\title{
Local recurrence rate in patients with DCIS
}

\author{
Bartova M, Suska P, Pohlodek K \\ IInd Department of Gynaecology and Obstetrics, University Hospital, Bratislava, Slovakia. \\ kamil.pohlodek@fmed.uniba.sk
}

\begin{abstract}
Objectives: The aim of this observational retrospective study was to evaluate the local recurrence rate of ductal carcinoma in situ of the breast (DCIS) and/or invasive breast cancer in patients with DCIS or microinvasive carcinoma of the breast after breast conserving or radical surgery. Secondary aim of the study was comprehensive assessment of the whole management of DCIS and its comparison with European guidelines. Methods: The study was performed in a group of 41 women with DCIS or microinvasive cancer, who underwent surgical treatment (breast conserving or radical modified mastectomy) at the IInd Department of Gynaecology and Obstetrics, University Hospital Bratislava (UNB), during the period 2001-2009. Documentation and pathological examination data from paraffin embedded tissue sections were used as data source. We sent out questionnaires regarding data about additional postoperative treatment and course of the disease up to year 2010 with focus on recurrence or tumour de novo incidence.

Results: Breast conserving surgery was performed in 28 cases $-68 \%$, modified radical mastectomy in 13 cases. All cases of mastectomy were due to multicentricity and/or extensive tumour $>4 \mathrm{~cm}$. Additional surgery due to unsatisfactory marginal status was performed in 8 patients (3.28\%). Additional treatment such as radiotherapy and/or hormonal therapy received 19 patients. Van Nuyss Prognostic Index was reported in 17 patients on the basis of histopathological data. 27 patients completed and returned questionnaire. No DCIS recurrence nor infiltrating cancer or tumour de novo was reported in this group.

Conclusion: We consider surgical management as adequate. Further material analysis is needed (Tab. 5, Fig. 1, Ref. 28). Full Text in free PDF www.bmj.sk.

Key words: ductal carcinoma in situ of the breast (DCIS), local recurrence, breast conserving surgery, margin status.
\end{abstract}

Ductal Carcinoma in Situ of the Breast (DCIS) represents an early form of breast cancer. It is defined as local carcinomatous disease confined to ductal system of the breast, with an absence of invasion to surrounding tissue. It is regarded an obligate direct precursor of invasive cancer of the breast. Its detection provides the possibility of less radical treatment of early- stage cancer with better prognosis and prevention from progression into invasive disease. DCIS represents a heterogenous group of lesions and since there is no universal marker indicating "DCIS-prone-to pogress", diagnosis of DCIS is always an indication for surgical intervention. An adequate diagnostic and surgical management should result in sufficient local control of the disease with prevention from the recurrence or progression. Concerning the fact, that there is only few comprehensive information regarding DCIS and its treatment in Slovakia, we decided to evaluate the management and course of the disease from the time of establishing the diagnosis up to

IInd Department of Obstetrics and Gynecology, University Hospital, Bratislava, Slovakia

Address for correspondence: K. Pohlodek, MD, PhD, IInd Dept of Obstetrics and Gynecology, University Hospital of Bratislava, Ruzinovska 6, SK-826 06 Bratislava, Slovakia.

Phone: +421.2 .948533504$

Acknowledgement: The authors thank their collegues from the Department of Pathology at University Hospital of Bratislava - Ružinov, Alpha medical pathology, s.r.o., and the Department of Radiology, University Hospital of Bratislava - Ružinov, for their cooperation and material support.
2010 (over the last 10 years). We assessed the material obtained at the IInd Department of Gynaecology and Obstetrics, University Hospital of Bratislava (UNB), with an average of 130 breast surgeries per year, 100 due to malignant disease.

\section{Methods}

This observational restrospective study was performed in a group of 41 patients diagnosed with DCIS or DCIS with microinvasion (defined as cancer with invasion within $1 \mathrm{~mm}$ (1) who underwent surgical treatment at the IInd Department of Gynaecology and Obstetrics, UNB during the period of 2001-2009. As a data source we reviewed documentation of the patients with the available post-operative pathologic data. Questionnaire concerning the course of the disease as well as historic data previous to the primary disease was sent out to all patients. The criteria for involvement in the study were following: surgical treatment at the IInd Department of Gynaecology and Obstetrics, UNB between 2001-2009, definite diagnosis of DCIS or microinvasive carcinoma of the breast from paraffin-embedded tissue sections, available documentation containing diagnostic and management data, pathologic results from the paraffin-embedded tissue sections, filled and returned questionnaire. The criteria for exclusion were: presence of invasive cancer or invasive cancer with extensive ductal in situ component (EIC) in paraffin embedded tissue, incomplete documentation, absence of the returned questionnaire/ inadequate answers in the questionnaire. 
Tab. 1. Assessed Data.

\begin{tabular}{|c|c|}
\hline Demographic data & age \\
\hline History data & $\begin{array}{l}\text { oncological disease in first-degree relatives } \\
\text { breast cancer in first-degree relatives } \\
\text { oncological disease in personal history } \\
\text { benign breast disease } \\
\text { irradiation of chest area } \\
\text { menarche } \\
\text { parity, age at the time of first labour } \\
\text { breast feeding } \\
\text { hormonal therapy }\end{array}$ \\
\hline Current disease & $\begin{array}{l}\text { manifestation of the diease } \\
\text { diagnostic management } \\
\text { surgical treatment } \\
\text { histopathological manner of the tumour (mor- } \\
\text { phology, margin status, extent, TNM classi- } \\
\quad \text { fication, VNPI) }\end{array}$ \\
\hline Questionnaire data & $\begin{array}{l}\text { postoperative treatment (RAT, CHT, hormonal) } \\
\text { recurrence/new tumour after primary therapy } \\
\text { (date of diagnosis, laterality, treatment, name } \\
\text { of the Workstation where tumour was diag- } \\
\text { nosed and treated) }\end{array}$ \\
\hline
\end{tabular}

VNPI - Van Nuyss Prognostic Index, RAT- radiotherapy, CHT - chemotherapy

Following data were assessed from the documentation (Tab. 1):

1. Demographic data: age of the patients.

2. Historic data: presence of cancer of any location in first-degree relatives and the age at which it was diagnosed, the incidence of breast cancer in first-degree relatives and age when diagnosis was established, malignant disease in personal history, incidence of a benign lesion of the breast in personal history (such as cyst, fibroadenoma, mastitis, other), previous irradiation of the chest area, menarche, parity, age at the time of the first labour, breast feeding span, hormonal therapy.

3. Data regarding current disease: clinical manifestation of the disease, diagnostic process, type and extant of surgical treatment, tumour characteristics (morphology, margin assessment, stage of the disease, Van Nuyss Prognostic Index (VNPI)).

Concerned Information within the questionnaire:

1. Historic information in order to confirm documentation data.

2. Course of the disease after surgical treatment: any subsequent therapy? - radiotherapy, chemotherapy, hormonal therapy (tamoxifen, other), name of the workstation where therapy was performed.

3. Incidence of any tumour that occured after the surgical treatment of in situ/microinvasive carcinoma at our department (recurrence or tumour de novo) - year of the occurrence, laterality (ipsylateral or contralateral breast with regard to primary tumour), name of the workstation where the tumour was diagnosed, applied treatment.
Tab. 3. Type of tumour.

\begin{tabular}{cccccc}
\hline DCIS & pT1mic & M.Paget & LCIS & Other & Overall \\
\hline 24 & 10 & 3 & 2 & 2 & 41 \\
\hline
\end{tabular}

DCIS - ductal carcinoma in situ, pT1mic - microinvasive carcinoma, LCIS - lobular carcinoma in situ

Tab. 4. Tumour manifestation.

\begin{tabular}{llr}
\hline Clinical and mammographic & palpable tumour & 7 \\
& nipple secretion & 2 \\
\hline Only on Mammography (Impalpable lesions) & microcalcifications & 27 \\
& shadow & 3 \\
\hline Only on Ultrasound & hypoechogenous lesion & 2 \\
\hline
\end{tabular}

\section{Results}

In the beginning of the study, 41 patients at the age of 25-68 years (average 56.69 yrs, median 53, modus 46 yrs) with the diagnosis of in situ carcinoma of the breast or microinvasive carcinoma 1-8 years after surgical treatment at our clinic were assessed (Tab. 2). According to the definitive post-surgical pathological assessment from paraffin-embedded tissue sections, in 24 cases a diagnosis of DCIS was established, 3 patients were diagnosed with lobular carcinoma in situ of the breast (LCIS) and in ten cases a microinvasive cancer (pT1mic) was reported. Within the group of DCIS, 8 carcinomas were identified as high grade DCIS with comedonecrosis, the rest accounted for low/intermediate DCIS or DCIS of unknown grade. Three cases presented with signs of M.Paget and in 2 cases pappilary noninvasive carcinoma was proved (Tab. 3 ).

Recurrence of the disease, 2 and 3 years after primary surgery performed out of our clinic, was suspected in 2 cases. Positive evidence of malignant disease in first-degree relatives was reporetd in 15 cases. In 3 of them cancer of the breast at the age of 54, 42 and 70 years occured. Benign disease of the breast was present in 11 women, 7 of them presented with benign cysts, 2 with fibroadenoma and 2 patients reported mastitis in their personal history. One patient mentioned other malignant disease in personal history (sigmoid colon cancer at 46 years of age, after surgical treatment). The average menarche age was 13.44 yrs (median 14, modus 14 years) the average parity rate 2.48 (median 2 , modus 2 ), average age at the time of the first labour 18.50 (median 22, modus 26) and average breast feeding lenghth was 5.96 months (median 4 months, modus 6 months). None of the patients had irradiation of the chest area during life. We noticed no significant correlation between the previous parameters and type of DCIS.

Clinical manifestation was noticed in 19 tumour cases. 17 patients had palpable tumour. From these, 2 were assessed as microinvasive carcinomas, 1 as high grade DCIS, the rest appeared

Tab. 2. Number of patients according to date of surgery.

\begin{tabular}{|c|c|c|c|c|c|c|c|c|c|}
\hline Year of treatment & 2001 & 2002 & 2003 & 2004 & 2005 & 2006 & 2007 & 2008 & 2009 \\
\hline Years after surgery to the time of analysis & 8 & 7 & 6 & 5 & 4 & 3 & 2 & 1 & \\
\hline Patients & 1 & 5 & 6 & 9 & 4 & 2 & 5 & 5 & 4 \\
\hline
\end{tabular}




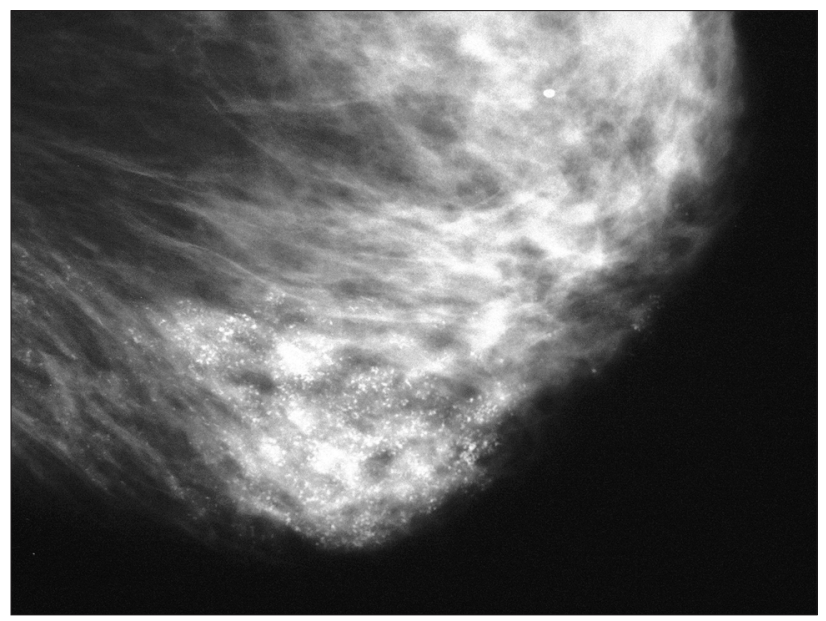

Fig. 1. Typical mammographic manifestation of DCIS with microcalcifications.

to be DCIS in the field of benign fibrodysplastic changes. Two tumours presented with sangvinolent nipple secretion, 2 patients with Morbus Paget noticed also inflammatory changes or mastodynia. Isolated changes on standard mammographic imaging were present in 30 cases. Microcalcifications were detectable in 27 of them, suspicious shadow was described in 3 cases. Abnormal changes of unspecified lesions were found only by ultrasound examination in 2 patients (Tab. 4 and Fig. 1).

All 41 patients underwent surgical treatment. 28 patients underwent breast conserving surgery (BCS). In 13 cases a modified radical mastectomy was performed. In all these cases mastectomy was due to multicentric disease or extent of the tumour. 6 cases of sentinel lymph node biopsy and 17 cases of axillary dissection were performed, all of them in cases, where characteristics of the disease indicated possibilty of invasive disease. The rest of the patients did not have axillary surgery. Additional surgery due to residual disease in surgical margins was performed in 8 patients. On the basis of data from the definitive pathologic evaluation we determined modified Van Nuyss Prognostic Index (age, extent of the tumour, grade, margin assessment) in 17 cases. 9 patients were referred to low risk group (VNPI 4-6), 7 patients to intermediate risk group (VNPI 7-9) and 1 into high risk group (VNPI 10-20) according to the counted VNPI. We were not able to count VNPI in the remaining patients for incomplete data in pathologic assessment.

Completed questionnaire was returned by 28 patients. 2 patients were additionally excluded from the group due to incomplete answers. Postoperative radiotherapy (RAT) reported 6 patients, in 6 women tamoxifen was administered postoperatively. In 7 patients combination of RAT and tamoxifen was used. 9 patients had no additional therapy, none of the patients received chemotherapy. In this group of patients, neither recurrence of the disease nor tumour de novo after the surgical tratment at out department occured.

\section{Discussion}

Aim of the surgical treatment of DCIS is an adequate removal of the tumour with tumour-free margins. The primary goal is to achieve balance between local control of the disease and good cosmetic result of the surgery. Current concept of the used treatment is based on thereview of few randomised trials evaluating various aspects of surgical and adjuvant treatment.

NSABP-B-06 (National Surgical Adjuvant Breast and Bowel Project) trial from 1976 is the only prospective randomised trial comparing modified radical mastectomy to breast conserving surgery with substantial radiotherapy (RAT) in patients with breast tumours no larger than $4 \mathrm{~cm}$ in the group of 1843 patients. Results show, that RAT following BCS may reduce recurrence rate by $40 \%$ in case of obtaining tumour free margins and is acceptable treatment for tumours in stage I and II of breast cancer.

NSABP-B-17 trial compared lumpectomy alone to lumpectomy with radiotherapy in patients with localized DCIS. Recently, updated results after 12 years support previous declaration of the study that radiotherapy can significantly decrease the rate of noninvasive and invasive recurrence (cumulative incidence of ipsilateral reccurence declined from $14.6 \%$ to $7.7 \%$ in radiotherapy group). Between 1991-1994 NSABP-B-24 trial evaluated 1804 patients divided into two groups, one with patients undergoing BCS followed by radiotherapy and 5 years of tamoxifen administration, the latter with placebo instead of tamoxifen. The recurrence rate of ipsilateral as well as contralateral cancer decreased significantly in tamoxifen group (2).

Despite constant efforts to find new ways of ,tailored treatment" (more or less demanding according to the type of tumour), at present treatment still follows mentioned results.

Following the European Guidelines for Quality Assurance in Breast Cancer Screening and Diagnosis (European guidelines), BCS should be performed as a standard treatment in $70-80 \%$ of patients with small MMG detected lesions. Modified radical mastectomy is appropriate therapy for larger tumours, in case of incomplete excision, where second surgery would result with poor cosmetic effect or in case of multicentric disease. Repeated surgery due to incomplete excision or not free margins should account for less than $10 \%$ cases. As for the axilla treatment, in true DCIS, the surgery of the axilla is not recommended. However, according to the German Association of Gynaecological Oncology (AGO), axillary staging in the mean of axillary dissection or sentinel lymph node biopsy is being considered in some cases of high grade, extensive DCIS with the possibility of microinvasion. As mentioned above, RAT after surgery may reduce the risk of recurrence by $40 \%$. Nevertheless, it has been proved, that effect is low in cases where tumour is smaller than $2 \mathrm{~cm}$, cases of low grade DCIS with tumour-free margins more than $10 \mathrm{~mm}$. According to published guidelines the mortality from DCIS should account for about $1.9 \%$ in 10 years. The mortality is probably associated with residual disease (of DCIS) and its progression into invasive cancer, presence of undetected invasive carcinoma at the time of diagnosis of DCIS or due to rise of de novo tumour (3). Of the prognostic factors such as age of the patient, extent of the disease, histologic grade ( higher progression risk in high grade DCIS), margin status seems to be crucial as far as the recurrence of the disease ( which is between 5-25\%) is concerned.

Considering previous recommendations, management of DCIS at our department consists of preoperative, operative and 
Tab. 5. AGO guidelines (7).

\begin{tabular}{|c|c|c|}
\hline & Oxford Level of Evidence & $\mathrm{AGO}$ \\
\hline Tumour-free margins in paraffin-embedded tissue sections (pR0) & $2 \mathrm{~b}$ & ++ \\
\hline Multifocal disease: limitid treatment possible in case of add. RAT & $2 \mathrm{~b}$ & + \\
\hline Additional surgery in case of close margins $(\leq 2 \mathrm{~mm})$ & $2 \mathrm{~b}$ & + \\
\hline Mastectomy in case of large tumours with uncertain margins & $2 \mathrm{a}$ & ++ \\
\hline SLNB ( $>5 \mathrm{~cm}$, DCIS, mastectomy) & $3 b$ & + \\
\hline Axillary dissection (axillary involvement due to undetected invasive disease only $2 \%$ ) & $2 \mathrm{~b}$ & -- \\
\hline
\end{tabular}

AGO - German Association of Gynaecological Oncology, add.RAT - additional radiotherapy, SLNB - sentinel lymph node biopsy, DCIS - ductal carcinoma in situ

postoperative measurements. Presurgical diagnostic management includes optimal imaging (mammography with magnification view if needed) followed by histological examination by ultrasound or stereotactic guided core biopsy. Prior to any surgery, in case of impalpable tumour, the lesion is localised by hook wire inserted into the breast (ultrasound or mammographic-guided insertion, as reffered elsewhere) (4). Next step is surgical management. Surgery should result in complete excision of the lesion with a tumour free margins. Since DCIS lesions are inappropriate for frozen sections, removal of the radiological lesion and approximate margin assesment is confirmed by specimen radiography, carried out intra-operatively by a radiologist. After ensuring that the whole radiological lesion has been removed, titanium clips are placed into the tumour bed to allow its accurate localisation. After receiving definitive histologic evaluation from paraffin-embedded sections, patient is reffered to the oncologist to discuss additional treatment. Clinical mammography is subsequently repeated.

Recently, the view at adequate surgical therapy of DCIS has changed in some points. BCS is based on the principle, that tumour-free margin is considered the most important predictor of local recurrence in DCIS. However, there is no consensus on what an adequate surgical margins refer to. According to Van Nuyss Scoring System designed by Silverstein, in the best prognosis group are those patients with clear margins defined as margins $>10 \mathrm{~mm}$. However, new noticable results regarding optimum margin status for BCS in DCIS were published recently. An analysis of published trials that examined outcomes after RT and BCS for DCIS was performed and data were extracted. Primary outcome was ipsilateral tumour recurrence rate related to surgical margin status. This meta-analysis included 4660 patients. Highest rates of ipsilateral breast tumour recurrence (IBTR) were observed with margins less than $2 \mathrm{~mm}$ compared to larger negative margins. However, further decrease in local recurrence was not observed when margins of 2 $\mathrm{mm}$ or more were compared with margins of $5 \mathrm{~mm}$ or greater $(5,6)$.

In recently updated recommendations, AGO supports the importance of tumour-free margins in correlation with local control of the disease and recommends additional excision in case of close margins, which are defined as margins $\leq 2 \mathrm{~mm}$ in paraffin-embedded tissue sections. AGO recommendations inculde also sentinel lymph node biopsy (SLNB) in DCIS $>5 \mathrm{~cm}$ or in case of mastectomy. It is possible to omit radiotherapy in already mentioned conditions (tumour $<2 \mathrm{~cm}$, margins $>10 \mathrm{~mm}$, low grade DCIS (7) (Tab. 5).

According to available literature data, DCIS accounts for around $10 \%$ of all detected carcinomas, in some USA countries even $20 \%$ (3). In Slovakia the incidence of DCIS is only $3.7 \%$ (8). In our study group, the annual average of DCIS ranged from 1 to 9 cases of DCIS (per 100 cases of malignant diseases of the breast), that means 1-9\%. In this group, limited surgery was performed in $68 \%$ cases (European Guidelines, $70 \%$ ). Additional surgery due to margin status was recorded in $3.28 \%$ of cases $(<10$ $\%$ according to the European Guidelines recommm). The number of recurrences as well as de novo tumours was 0 .

According to these data we consider the surgical management as adequate. We would like to continue with the material analysis. We are also aware of the need of thorough and consistent data aquisition in order to increase the quality and number of the material.

\section{Conclusion}

Regarding increasing incidence of early forms of breast cancer thanks to mammographic screening in clinically asymptomatic patients, the value of the diagnosis and its subsequent therapy is obvious. DCIS is a relatively new notion among the diagnoses of breast diseases and an adequate treatment is a commonly discussed problem. DCIS represents a heterogenous group of lesions, which are proved to be obligate direct precursors of invasive cancer. The apropriate therapy with obtaining tumour-free margins should lead to local control of the disease in sense of better prognosis (prevention of systemic disease) and should shift the therapy of breast cancer into its early stages. It offers the possibility of less radical and mutilating treatment-BCS (if the extent and localisation of the disease enable it). Thorough evaluation of the management and further course of the disease is control of adequate practice in accordance with available knowledge. Integrated processing of the practice makes it possible to record any sequences and mistakes, that might be overlooked. It enables to improve the management, follow the trends of the disease in our country as well as confrontation with world-wide trends. Good example of the changing trends is also information on adequate margin status in correlation with recurrence rate after DCIS or new ways of thinking about RAT and axilla treatment.

\section{References}

1. Perry N, Broeders M, Wolf C, Törneberg S T, Holland R, Karsa L. European guidelines for quality assurance in breast cancer screening and diagnosis. European Communities 2006: 259-267, 325-331.

2. http://www.nsabp.pitt.edu

3. Tavassoli FA. Ductal intraepithelial neoplasia of the breast. Virchows Arch 2001; 438: 221-227. 
4. Pohlodek K. Mamografická stereotaxe a intervenční výkony. 35-51. In: Daneš J et al. (Eds). Základy mamografie. Praha: X-Egem, 2002.

5. Pleijhuis R G, Graafland M, Vries J, Bart J, Jong J S, Dam G M. Obtaining adequate surgical margins in breast-conserving therapy for patients with early-stage breast cancer: Current modalities and future directions. Annal Surg Oncol 2009; 16: 2717-2730.

6. Olson TP, Harter J, Munoz A, Mahvi DM, Breslin TM. Frozen section analysis for intraoperative margin assessment during breast-conserving surgery. Results in low rates of re-excision and local recurrence. Annal Surg Oncol 2007; 14: 2953-2960.

7. http://www.ago-online.de

8. http://www.nczisk.sk

9. Dunne C, Burke JP, Morrow M, Kell MR. Effect of margin status on local recurrence after breast conservation and radiation therapy for ductal carcnoma in situ. J Clin Oncol 2009; 27: 1615-1620.

10. Daneš J, Skovajsová M, Bartoňková $H$, Svobodník A, Krejčová I. Screening karcinomu prsu v České republice. Současnost a budoucnost, úloha gynekologa. Karcinom prsu. Moder Gynekol Porod 2004; 13: 464-476.

11. Pavlišta D et al. Neinvazivní karcinomy prsu. Praha: Maxdorf, 2008: $181 \mathrm{p}$.

12. Fonseca R, Hartmann LC, Petersen IA, Donohue JH, Crotty TB, Gisvold JJ. Ductal carcinoma in situ of the breast. Annal Intern Med 1997; 127: 1013-1022.

13. Morrow M. Understanding ductal carcinoma in situ. A step in the right direction. Cancer 1998; 86 (3): 375-377.

14. Rebner M, Raju U. Noninvasive breast cancer. Radiology 1994; 190: 623-631.

15. National Institutes of Health State-of-the-Science Conference Statement. Diagnosis and management of ductal carcinoma in situ (DCIS). Draft Statement September 24; 2009.

16. Bornstein BA et al. Results of treating ductal carcinoma in situ of the breast with conservative surgery and radiation therapy. Cancer 1991; 67: 7-13.
17. Swain SM. In situ or localized breast cancer - how much treatment Is needed? New Engl J Med 1993; 328: 1633-1634.

18. Schnitt SJ, Harris JR, Smith BL. Developing a prognostic index for ductal carcinoma in situ of the breast - are we there yet? Cancer 1996; 77 (11): 2189-2192.

19. Geza Acs. Ductal carcinoma in situ: Current concepts. Patologia 2010; 48: $180-193$.

20. Mulcahy N. Take carcinoma out of DCIS and ease off treatment. J Natl Cancer Inst 2010; 102: 1-9, 170-178.

21. Fisher B et al. Lumpectomy compared with lumpectomy and radiation therapy for the treatment of intraductal breast cancer. New Engl $\mathrm{J}$ Med 1993; 328: 1581-1586.

22. Veronesi $\mathbf{U}$ et al. Radiotherapy after breast-preserving surgery in women with localised cancer of the breast. New Engl J Med 1993; 328: $1587-1591$.

23. Lulien JP et al. Radiotherapy in breast-conserving treatment for ductal carcinoma in situ: first results of the EORTC randomized phase II trial 10853. Lancet 2000; 355: 528-533.

24. Young O, Valassiadou K, Dixon M. A review of current practices in breast conservation surgery in UK. Ann R Coll Surg Engl 2007; 89: $118-123$.

25. Fisher B et al. Tamoxifen in treatment of intraductal breast cancer: National Surgical Adjuvant Breast and Bowel Project B-24 randomised controlled trial. Lancet 1999; 353: 1993-2000.

26. Wärnberg F, Nordgren $\mathbf{H}$, Bergh J, Holmberg L. Ductal carcinoma in situ of the breast from a population-defined cohort: an evaluation of new histopathological classification systems. Eur J Cancer 1999; 35 (5): 714-720.

27. Thomssen C. Aktuelle Empfehlungen zur Therapie primärer und fortgeschrittener Mammakarzinome. State of the Art 2007: 69-84.

28. Fisher B, Dignam J, Tan-Chiu E et al. Pathologic findings from the National Surgical Adjuvant Breast Project (NSABP) eight-year update of protocol B-1. Intraductal carcinoma. Cancer 1999; 86: 429.

Received January 26, 2011. Accepted September 20, 2011. 